\title{
Delineating the Model-Stakeholder Gap: Framing Perceptions to Analyse the Information Requirement in River Management
}

\author{
Judith A. E. B. Janssen • Arjen Y. Hoekstra • \\ Jean-Luc de Kok • Ralph M. J. Schielen
}

Received: 9 July 2007 / Accepted: 1 September 2008 /

Published online: 8 October 2008

(C) The Author(s) 2008. This article is published with open access at Springerlink.com

\begin{abstract}
Computer models can support policy development in environmental management, but often suffer from a lack of practical application. This is part due to a 'gap' between the ways in which various participants in the policy process deal with information. The framework described in this paper provides a structured approach to information analysis in policy processes. The awareness of differences in the nature of information helps in dealing with different perceptions and actor backgrounds during this policy process because it allows a better match between the information needs and the tools or procedures that are applied. Construal level theory, originating from consumer psychology, can account for some differences that remained unaddressed so far and forms a key component of this framework. Application of the framework to the Dutch Maas case shows that a gap between the model and its users is unavoidable; model applications are dominated by the fairly technical conceptions which modelers have of the system, and fail to address high level construals brought up by the stakeholders. Application of the framework can support the match between mutual expectations of modelers and users.
\end{abstract}

Keywords Policy analysis • Perceptions • Construal level theory $\cdot$ River modeling • Stakeholder participation $\cdot$ River Maas

\section{Introduction}

Computer models can support policy development in environmental management, owing to their ability to allow for complex calculations and to process large amounts

J. A. E. B. Janssen $(\bowtie)$ - A. Y. Hoekstra · J.-L. de Kok

Water Management and Engineering Group, University of Twente,

P.O. Box 217, 7500 AE, Enschede, The Netherlands

e-mail: j.a.e.b.janssen@utwente.nl

R. M. J. Schielen

Rijkswaterstaat Waterdienst, Lelystad, The Netherlands 
of data. However, many computer models suffer from a lack of practical application, despite the financial, human and technical resources that are spent (Walker 2002). The benefits of computer support for policy making repeatedly turn out to be smaller than expected. The limited application of computer models or decision support systems is attributed to a gap between the model makers, experts and/or researchers on the one hand, and stakeholders, policy makers and/or end-users on the other (Olsson and Anderson 2007; Brugnach et al. 2006; Borowski and Hare 2007; De Kok and Wind 2003). This particularly plays a role in the early stages of the policy cycle, where models may be used as eye-openers, as tools to solve dissent in the process, or as tools used to arrive at consensus (Van Daalen et al. 2002). The gap between different parties in the policy process partially lies in the way in which they perceive the information requirements. Different perceptions of the problem lead to different foci in the policy process. A tighter connection between these should help improve the use of models and the use of model results in the policy process. Suggested solutions to achieve this are the improvement of communication on the expectations people have from the models, and assumptions underlying them, and the early involvement of stakeholders or policy makers in the model building process (Otter et al. 2004; Pahl-Wostl 2002; Brandon 1998). Still other studies show that a tension remains between the availability of human and technical resources, and the complexity and coherence of the real world, as it is increasingly communicated by stakeholder participation (Matthies et al. 2007). What is modeled usually depends on data and model concept availability, and this is not always sufficient for making a decision. Many recommendations in the modeling literature hence aim at providing guidelines for optimizing the 'return on investment'; the tests of relevance, measurability, data-availability and simplicity are guiding to the development of indicators for modeling (Nieuwkamer 1995; World Bank 1999; Lorenz et al. 2001; Niemeijer 2002; Dale and Beyeler 2001). Providing the right information in the right amount, without being all too comprehensive, is the general guideline in modeling.

While aiming to build a quick scan tool for river management in which the indicators (i.e. model output) are based on stakeholders' information needs, we came across the problems described above. Using the stakeholders' contributions to the policy process in a model tool requires a justification of the choice of indicators taken into account, and a clarification of the extent to which we think we can contribute to overcoming the gap between the model and its user. Until now, this topic is addressed either from the modeler perspective or from a more 'process-oriented' perspective. The first putting forward arguments such as data availability, measurability and so forth, and the second focusing more on the role of power, behavior and interests. Both do not explicitly address the question of why the criteria or indicators (used in models and by stakeholders) themselves differ, and why, and to which extent, certain criteria appeal more to stakeholders. To gain a better understanding of the gap between models and stakeholders' perceptions a more in-depth examination of the reflection of different perceptions in information in the policy process is required. By describing the assessment indicators used by stakeholders, and comparing these to modeling requirements such as data and model availability, the gap between the modelers' perceptions and the stakeholder perceptions can be better anticipated in an early stage of the modeling process. In this way, different tools and methods used 
in policy processes can be matched with the information needs during this process. The 'gap' is explicitly not addressed as a difference between information supply and demand; Turnhout et al. (2007) demonstrate that all parties involved may offer and require information throughout the process. Moreover, these may affect each other which leads to a web of information in which supply and demand are hard to disentangle.

We use 'indicators' (or 'decision criteria' or 'decision variables') as our unit of analysis. They are considered to represent the information in the policy process. The hypothesis of this study is that the emergence of a 'gap' is inevitable, but that an appropriate description of the nature of indicators used in a policy process can explain part of the gaps' origin and help directing the model effort. In order to close the gap, an interdisciplinary approach needs to be adopted. A single viewpoint will not suffice to account for the differences in people's perceptions and ways of working.

A framework is developed that addresses the differences between indicators originating from different perceptions. We observe differences in temporal scale, spatial scale and the represented river function. Yet these properties don't appear to account for all the differences we found. Crucial in this framework is therefore the addition of the construal level as one of the dimensions of indicator assessment. Construal level theory originates from psychological science, and offers an account of how psychological distance influences peoples' thoughts and behavior (Trope et al. 2007). It helps explaining why, for instance, flood catastrophes which only rarely occur and receive little attention in the media until they do, are usually described in rather general terms by stakeholders. Together with the other characteristics construal level theory can help explain why the information supplied by models sometimes does appeal to its end-users, and sometimes it does not. It helps identifying 'blanks' in the information space, for which other methods than modeling such as stakeholder or expert consultation need to be applied.

The research approach, outlined in Fig. 1, is complementary to social learning and participatory modeling approaches (Pahl-Wostl 2002; McLain and Lee 1996). The latter focus mostly on the process and the role of the model in it, whereas this paper focuses on the content of both the model and the policy process in which it is applied.

Section 2 gives an introduction of the case study that is used to test the framework. The case study concerns the Explorative Study of the river Maas (in Dutch denoted IVM; this abbreviation will be used in the remainder of this paper; Ministerie van Verkeer en Waterstaat and Rijkswaterstaat Dienst Limburg 2003). In this project models were used and stakeholders were consulted for the assessment of different river management strategies. Section 3 outlines the framework used to compare the perceived information requirements by modelers and stakeholders. It categorizes the information requirements into different river functions, and next shows how information can be characterized based on temporal and spatial scale and the level of construal. The results of applying this framework to the case study are described in Section 4. They consist of a typology of both model and stakeholder indicators in the light of the framework presented in Section 3, and the comparison of both based on this framework. The last section contains a number of conclusions drawn from the development and application of the information typology framework. 
Fig. 1 Research approach

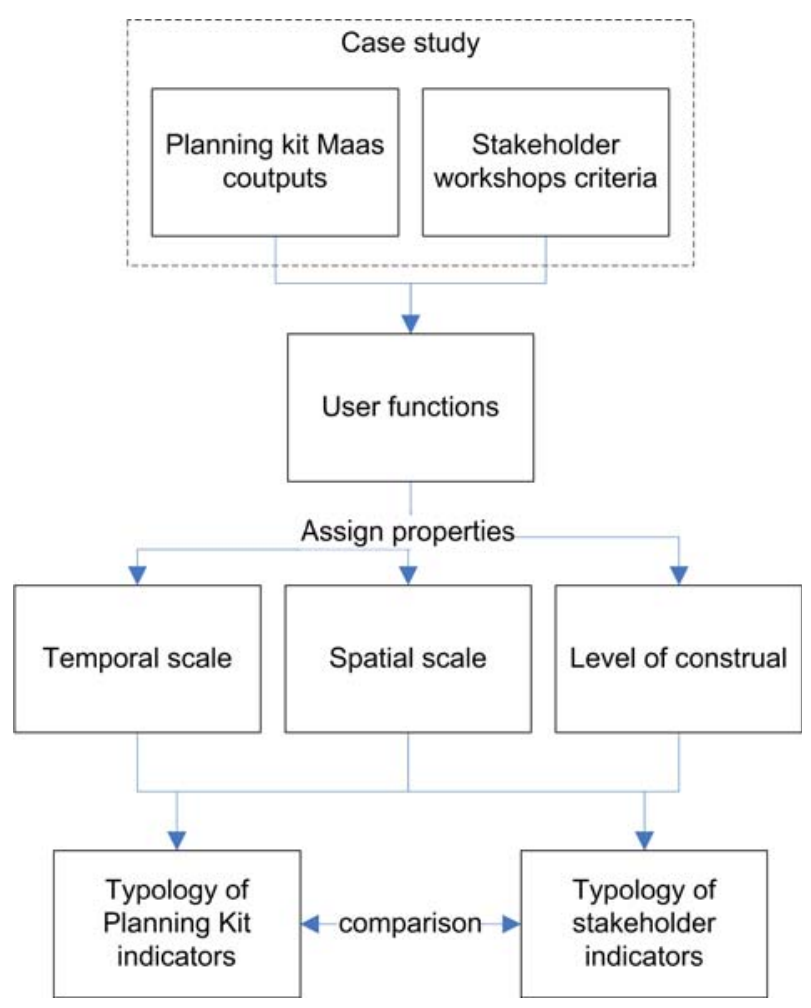

\section{Case Study: The Integrated Explorative Study of the Maas}

The framework, developed in the next section, is applied to the IVM study. In this study a model was used in a stakeholder setting, which means that both model and stakeholders were in the process at the same point. This assures a fair as possible comparison. The study concerns future flood safety along the river Maas.

\subsection{The River Maas}

The Maas is the Dutch stretch of the French/Belgium/Dutch river Meuse. The river originates in the north of France. It flows through France, Belgium and the Netherlands before it discharges into the North Sea. The total river basin area, is approximately $36.000 \mathrm{~km}^{2}$, of which about $7.700 \mathrm{~km}^{2}$ is located in the Netherlands (Fig. 2). The Dutch part of the Maas has a length of almost $300 \mathrm{~km}$ (Ministerie van Verkeer en Waterstaat and Rijkswaterstaat Dienst Limburg 2003). Of the Dutch population, 22\% lives in the Maas catchment (Busch 2004). In the South of Limburg the urban land-use adds up to about $20 \%$ of the catchment area, further downstream this reduces to about 10\% (Busch 2004). About $65 \%$ of the area in the catchment has an agricultural function.

Because of the relatively small rain-fed catchment, the Maas discharge strongly responds to rainfall. Rainfall averages $40 \mathrm{~mm} / \mathrm{month}$ in dry months and $74 \mathrm{~mm}$ in 
Fig. 2 The river Maas catchment [www.RIWAMaas.org]

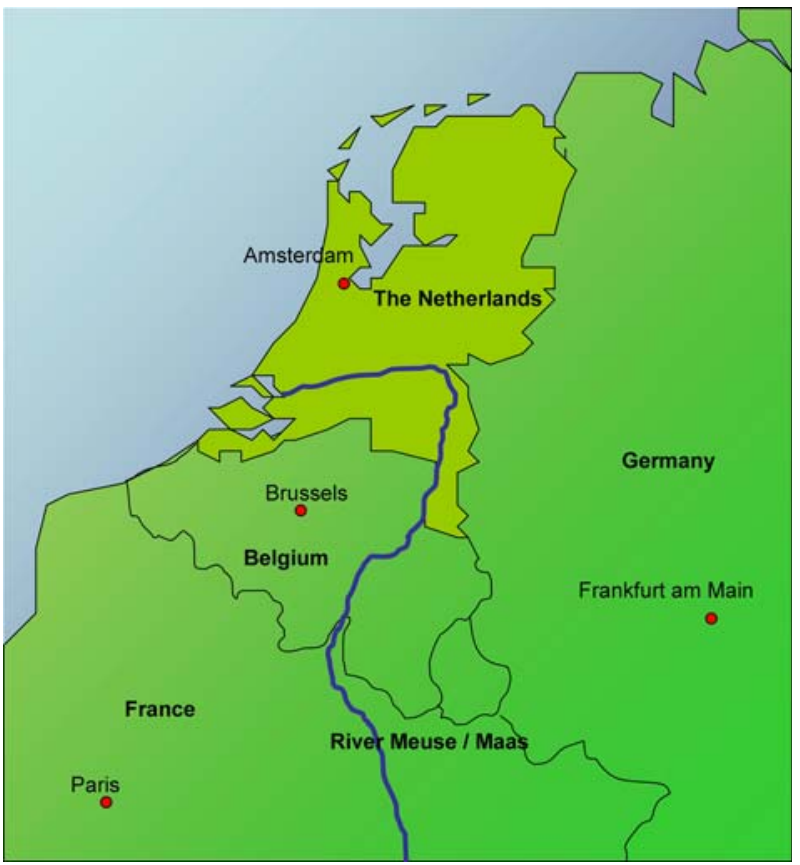

wet months. Taking a whole year into consideration, there is more precipitation than evaporation and the precipitation balance turns out positive (Busch 2004). The strong response of the discharge to precipitation is partially due to the natural shape of the river basin, which is rather deep incised and, particularly in the upper part of the catchment, consists of impermeable soil. Another contribution to the quick discharge of precipitation are the narrow floodplains, created as a result of river canalization, particularly in the Belgian and Dutch part of the river.

Along the Dutch Maas, two situations are distinguished. In the upstream part the Maas flows through a v-shaped valley, where there is no large scale protection by dikes. The terrace landscape in which the river is embedded, is the result of erosion during the ice age (Ministerie van Verkeer en Waterstaat and Rijkswaterstaat Dienst Limburg 2003). In this part gravel excavation takes place. Due to the steep slopes of the banks flooding depths in this part remain limited, and the flooding probability is-compared to other Dutch flooding probabilities-relatively high. At several locations different functions such as urban settlement, flood protection and nature development, are combined in the winter bed. Local municipalities are protected by artificial levees. The downstream part of the river flowing through the Dutch lowlands, has artificial levees to protect the hinterland. Flooding of the diked area did not occur during the past decades. Embankments have lowered the flooding probability to 1:1250 years, but simultaneously increased inundation depths behind the dikes in case of a flood. In the river mouth various structures have been built to protect the land situated below sea-level from flooding by both the river and the sea. In the upstream part of the river, flooding occurred in 1993 and 1995. 


\subsection{The IVM Project}

In 1993 and 1995 the Netherlands were confronted with high discharges on the Maas and Rhine branches and consequent flooding in the upstream part of the Maas. Although there were no casualties, the large damage and the prospect of more high discharges in future due to climate change urged for measures, something that was felt by inhabitants of the area as well as local, regional and national governments.

The threat of an increase in future peak discharges led to a series of three explorative studies on the Maas. Wesselink (2006) gives a detailed overview of the policy planning process. The first study was the Explorative study on Expansion of the Maas cross-section (Dutch: Verkenning Verruiming Maas, VVM). The objective of this study was to formulate strategies to maintain the current maximum water levels even when the discharge would increase to $4.600 \mathrm{~m}^{3} / \mathrm{s}$. This discharge corresponds to the worst climate change scenario for 2050 or to the average scenario for 2100 Ministerie van Verkeer en Waterstaat and Rijkswaterstaat Dienst Limburg (2003). Currently, the maximum conveyable discharge (design discharge) is $3.800 \mathrm{~m}^{3} / \mathrm{s}$. The VVM-study was a hydraulic study in which the effects of climate change were calculated, along with the measures that could be taken to mitigate these effects. The study turned out to be not sufficient to allow for a choice of measures, and hence got a follow-up. Its successor was IVM-1 (Integrated Explorative study of the Maas). In this study, politicians, civil servants, and interest-organizations were represented in three different working groups and invited to contribute to the discussions. The discussions were widened into other fields than merely hydraulics. Eventually sets of measures were composed based on two principles: spatial quality and future development scenarios. During it's follow-up in IVM-2 particular attention was to be given to the 'opinion of the region'. The concrete translation of the IVM-2 assignment was to 'design a broadly supported set of measures that a) provides safety and b) contributes to spatial quality' (Wesselink 2006). To incorporate the opinion of the region IVM-2 used three series of one-day workshops. The 'region' was defined by splitting up the Maas trajectory in four parts; two upstream and two downstream. The three workshops were held in the four distinguished Maas trajectories, so a total of 12 workshops were held. In these workshops the proposed measures, resulting from IVM-1, were discussed. Besides the representatives from the groups that participated in IVM-1, also representatives of communities, water boards and additional interest groups were invited for IVM-2. Among the latter were nature organizations and people representing recreational and industrial interests. It is from these workshops that we derived the material used in this study. The stakeholders' ideas and opinions, expressed during the workshops, are in this study compared to a policy support tool that was used from the second series of workshops onwards. This tool is essentially a database comprising the results of the first IVM study and is known as the Planning Kit Maas (developed by WL Delft | Hydraulics). It provides scores of the different proposed river reconstructions measures on a number of objectives. After the second series of workshops experts had to work on the sets before these sets would eventually meet the objective of both safety and of spatial quality. In the third and final series of workshops the resulting sets of measures, which solved the hydraulic problem and had sufficient social support, were presented to the workshop participants, and the process was evaluated. 
We used both the stakeholders' arguments and the Planning Kit Maas, both as applied in IVM-2, to obtain the data used in this paper.

\section{Framework: Describing the Nature of Information}

In this study, the type of information provided by the Planning Kit Maas will be compared with the type of information that stakeholders used in the discussion about the different management alternatives. For this comparison between model and workshop a framework was used in which we distinguish between four features of information:

1. River function to which the indicator is linked;

2. Temporal scale of the process to which the information refers;

3. Spatial scale of the process to which the information refers;

4. Level of construal of the information. The level of construal refers to a continuum from concrete to abstract. Concrete pieces of information are low-level construals, abstract pieces of information are higher level construals. According to construal level theory (CLT), the psychological distance (social, temporal, spatial and hypothetical distance) relates to the way in which people perceive things and to the way they decide about things (Trope et al. 2007). The following subsections elaborate this framework.

The first three are frequently used throughout literature as a basis for indicator development or description (e.g. Gibson et al. 2000; De Groot 1992). However helpful they turned out to be insufficient to account for some differences in the nature of indicators used by modelers and stakeholders. The construal level originates from consumer psychology and proves to add a helpful dimension to the analysis and to provide additional insight in the different perceptions.

\subsection{River Functions}

As described by Pahl-Wostl (2004), integrated assessment involves multiple tradeoffs. Classification of the different trade-offs forms the first step in the comparative framework. Generally the trade-offs concern different stakeholders, proceeding from their respective interests. The stakeholders' objectives reflect these interests. The objectives and interests depend on the stakeholders' roles in the environmental system or, in other words, on the functions they utilize in the system. A similar line of reasoning is comprehensively elaborated on by De Groot (1992). He defines ecosystem functions as '...the capacity of natural processes and components to provide goods and services that satisfy human needs, directly or indirectly'. The concept of ecosystem goods and services is inherently anthropocentric; it is the presence of human beings as valuing agents that enables the translation of basic ecological structures and processes into value-laden entities. This value need not necessarily be monetary. The four main categories of functions distinguished by De Groot (1992) are

- Regulation functions (e.g. regulation of run-off, maintenance of biodiversity);

- Carrier functions (e.g. agriculture, shipping); 
Table 1 River functions

\begin{tabular}{ll}
\hline Type & Function \\
\hline Regulation & Regulation of run-off and flood protection \\
& Water catchment and groundwater recharge \\
& Prevention of soil erosion and sediment control \\
& Storage and recycling of human waste \\
& Maintenance of biological and genetic diversity \\
& Human habitation and settlements \\
Carrier & Cultivation/agriculture \\
& Recreation and tourism \\
& Nature (protection) \\
& Infrastructure \\
& Landscape \\
& Navigation \\
Production & Water (cooling, drinking, regional water supply) \\
Information & Providing historic information \\
\hline
\end{tabular}

- $\quad$ Production functions (e.g. raw materials, drinking water);

- Information functions (e.g. aesthetic information, historical information).

A number of sub-functions identified by De Groot (1992) apply to river systems. These are listed in Table 1. The stakeholders' arguments and model outputs are all assigned to one of these functions. The overview of sub-functions can also be used to examine the objectives in a certain management problem categorically, or to explore the stakeholder participation. It gives a general starting point to environmental problem explorations.

\subsection{Temporal Scale}

The second characteristic of information used in the proposed framework is the temporal scale of the physical process underlying the indicator. Many scientists have acknowledged the relevance of scaling issues in integrated modeling, a key aspect of the integration between social and natural sciences (Gibson et al. 2000). Van der Veen and Otter (2003) note that '...choosing a scale on which to project the objects and processes in a model refers to a quantitative and analytical dimension and to time and space.' Characteristic time scales of a process can be defined as (a) the lifetime or duration of the process, (b) the period or cycle for periodic processes, or (c) the correlation length or integral scale (Blöschl and Sivapalan 1995). In the following, the 'period or cycle' of a certain process is referred to when talking about the temporal scale. As Evans et al. (2003) conclude, it would be ideal to analyze processes along a continuum of scales rather than at a certain point of a given scale. However, this is not practical due to data availability issues and computational limitations. Also for the sake of comparison between two datasets, a continuum is not optimal. Therefore three levels of the temporal scale are distinguished here. The shortest time scale involves processes taking place over days or months (or shorter), such as the morphological changes due to peak discharges or the peak discharges themselves. For river management, it is not necessary to look at timescales of seconds or minutes, as applying to for instance turbulence. The medium time scale involves processes taking place over several years. The long time scale concerns slow processes such as morphological changes in river inclination, taking place over decades. An important 
remark to be added here is that not all decision criteria depend on processes. The 'stability of the current dikes' is an example of a variable that affects the decision and represents the 'status quo', rather than being process-dependent. Variables like these will be assigned to a fourth class in which no specific time-scale applies; schematized along the zero of the temporal axis.

\subsection{Spatial Scale}

Spatial scale has the same acknowledged relevance to modeling as temporal scale. Also in spatial scale a distinction can be made between the spatial extent of a process, the period and the integral scale. Again here, the 'period of the process' is considered to determine the spatial scale; i.e. the area over which a process cycle can be measured. According to Blöschl and Sivapalan (1995), '...scale refers to a rough indication of the order of magnitude rather than to an accurate figure'. Again, a distinction is made between three categories. In different categories, different types of processes dominate. A small scale is considered to concern processes that are described on an extent of 10-100 m, for instance the morphological processes of small bed forms. A medium spatial scale refers to processes taking place on a scale of 100-1000 m, such as agriculture, or the effect on geological values. Scales exceeding several kilometers, and hence involving a large part of the catchment, are classified as large scale processes or indicators. For river management, the spatial scales ought to be regarded relative to the size of the catchment under study.

\subsection{Level of Construal}

Not all the differences in the nature of information can be accounted for by looking at temporal and spatial scales and river functions. There is also a difference in the way in which stakeholders and modelers construct information. In the IVM case, where the two points of view are confronted in a workshop process, this was observed very clearly. The modelers tended to focus more on the details and technical and specific features of measures or effects; attributes that only have a value when placed in the context of a particular location and measure. The stakeholders on the other hand, tended to discuss the problem in a more general and decontextualized sense, while at the same time addressing the proposed measures in a more detailed and specific manner. Framing these differences implied having to look for an extension of the theoretical framework. Construal Level Theory, or CLT, originating from consumer psychology, offers this extension. Moreover, it also offers an explanation of what is underlying the observed differences. Psychological construal level theory (Liberman and Trope 1998) offers more grip on the nature of information in general, in this case applied to indicators used in river management. The construal level links events that happen more often, to a more detailed, precise and accurate description than events that are less likely to happen (Wakslak et al. 2006). Wakslak in particular builds a link between the level of construal and probability. A higher construal level (i.e. a development that is further away in time, space, or social distance) leads people to describe things in a more generic and less detailed manner. High level construals are '...decontextualized representations that extract the gist from the available information. These construals consist of superordinate, general and core features of options. Low-level construals are less schematic, more contextualized 
Table 2 Description of high and low construal levels

\begin{tabular}{ll}
\hline High level construal & Low level construal \\
\hline Distant in time, space or social environment & Near in time, space or social environment \\
Superordinate goals & Subordinate goals \\
Categorization leads to few broad classes & Categorization leads to many narrow classes \\
Abstract & Concrete \\
Decontextualized & Contextualized \\
Example for water management: Safety & Example for water management: water level \\
\hline
\end{tabular}

representations of information about options. These include subordinate, specific and incidental features of options. For example, a high-level construal may represent 'moving into a new apartment' as 'starting a new life', whereas a low-level construal may represent the same event as 'packing and carrying boxes.' (Trope 2004). Further, CLT proposes that '... the same information is construed at a higher level when the information pertains to distant-future events than when it pertains to near-future events.' (Trope 2004).

The differences in construal levels are attributed to the relationship between direct experience and information about an event. Typically, as an event becomes removed from direct experience (e.g. as an event is placed further into the future), information about the event becomes less available or reliable, leading individuals to form a more abstract and schematic representation of the event. Later researchers have argued that this distance need not necessarily be a matter of time, but could also arise in space or social distance (Wakslak et al. 2006; Trope 2004; Liberman and Trope 1998). The general characteristics corresponding to high and low levels of construal are summarized in Table 2.

The level of construal shows parallels with the level of analysis as described by, for instance, Van der Veen and Otter (2003). They however mostly refer to aggregation in the model, and hence make a direct link to temporal and spatial scale, whereas the level of construal rather relates to peoples' perceptions of the phenomenon under study. It gives information not only about the scales at which the physical processes take place, but also about peoples' perceptions of them. For the level of construal, a distinction is made between a low level of construal (concrete indicators, contextualized and specific information), intermediate level of construal (indicators that are in between the other two) and high level of construal (superordinate, general, core features of options). In river management, a high construal level indicator would be 'safety', and its low level construal counterpart 'water level decrease following a certain measure in cm'. The former is general, decontextualized and superordinate, whereas the latter is subordinate, contextualized (i.e. only meaningful when considered in a specific context) and has a high level of detail.

Summarizing, the comparison is based on a distinction in river functions and, for every one of these, a score on three dimensions:

1. Temporal scale

2. Spatial scale

3. Level of construal

For all three of these, a distinction in three classes is used. Graphically the framework can be depicted as shown in Fig. 3, for every function or sub-function set. 


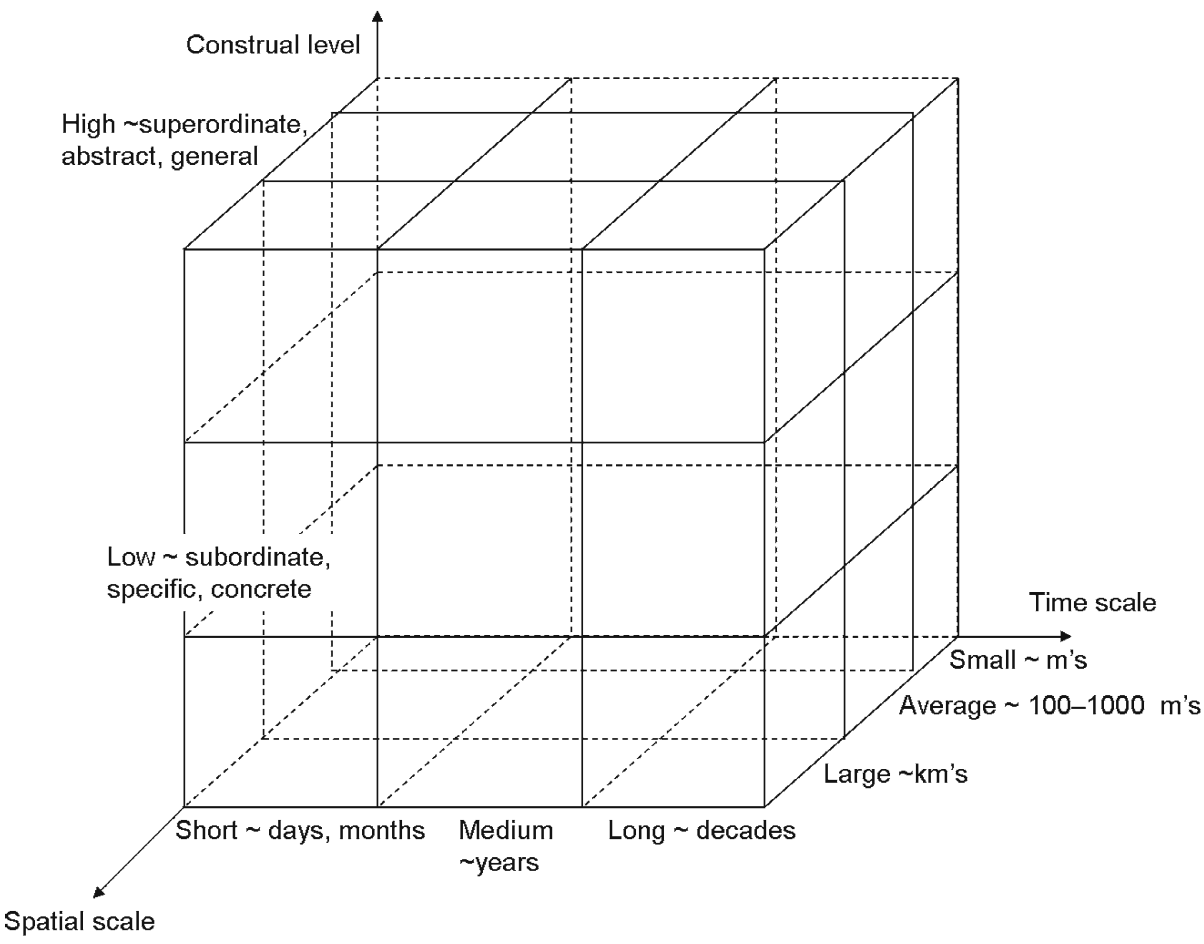

Fig. 3 Information typology: categories for river management

\section{Results}

With the help of the framework described in the previous section the output indicators of the Planning Kit Maas are compared with type of information used in the arguments of workshop participants. For both, the context is outlined first, followed by a description of how the framework was applied.

\subsection{Indicators Resulting from the Planning Kit Maas}

The Planning Kit Maas is a database tool in which knowledge from various sources has been collected. The indicators concerning the rivers' discharge function originate from a schematization of the river Maas with the water-flow model SOBEK® by WL Delft Hydraulics. For the functions 'agriculture' and 'habitation and settlements' the outcomes in the Planning Kit originate from map comparisons, while for landscape quality the effects were discussed in expert groups. The outcomes were reported for every measure separately. This means that extensive discussion about which indicators are relevant for measure assessment, already underlies this model. Additional information to this model was available in other studies, providing the experts with a good insight in the technical aspects of the different measures. In that respect there was a large gap between the knowledge that had been previously generated and collected during the project (i.e. the knowledge of most experts in the project), and 
the knowledge of the workshop participants. Yet, the workshop participants still had some contributions to make to the evaluation of the different river strategies.

An overview of the assessment of model outputs is shown in Table 3. In the light of the functions from Table 1, indicators were found for the functions 'regulation of run-off and flood protection', 'human habitation and settlements', 'cultivation/ agriculture', 'nature', 'landscape' and 'provide historic information'.

Two examples are elaborated to demonstrate the framework's application; the indicators relate to the river functions of 'regulation of run-off and flood protection' and 'nature'.

\subsubsection{Regulation of Run-off and Flood Protection}

- Recurrence probability: is determined on a large time scale, in this case 250 and 1250 years for the undiked and diked area, respectively. It features a large spatial scale, since the catchment and catchment precipitation determine the discharge. Further, the recurrence probability can be seen as a very concrete and specific property of an extreme flood event, and is therefore a low level construal.

- Total decrease of the water level in centimeter: follows from a comparison of the maximum water level in the current situation compared to that after measures have been implemented. The water levels are calculated based on peak discharges, taking place in cycles of a couple of days. The temporal scale is small. The decrease of water levels depends on the location and type of measure, and the length of backwater curves. Taking the geographical size of the catchment into account, a medium spatial scale is assigned. The decrease of the water level in centimeter depends on local circumstances and is meaningless without this context. It is also a subordinate variable, and considered a low level construal.

- Design water level gain: derived from the previous indicator, hence assigned the same characteristics.

- Change in the discharge peak and front shape: both relate to traveling of the discharge peak through the catchment and the amount of water. This process is usually described over a period of several days; a small temporal scale applies. The spatial scale is large, because a large part of the catchment has to be taken into account. Again the variable is described on a low construal level.

- Levee construction: refers to the kilometers of levee required for a set of measures. This is a static indicator linked to individual measures. Due to this static nature, a small temporal scale is assigned. Because it is linked to individual measures, and the implementation strongly depends on local landscapes, an average spatial scale applies. Again, it is a concrete representation of a specific property of the measure, and a low construal level applies.

- Investment cost: relates to individual measures, similar to the previous.

- Management and maintenance cost: applies to individual measures, but can only be calculated by taking a longer period into account. A medium spatial scale applies, in combination with a large temporal scale, to capture the life-span of the measure. For construal level the same applies as for the levee construction and investment cost, resulting in a low level of construal.

- Total cost: cumulative variant of the previous. Because it is cumulative, it requires a long time horizon (because maintenance is also taken into account) and a large spatial scale (because all measures in the catchment are considered 
Table 3 Typology of indicators in IVM case

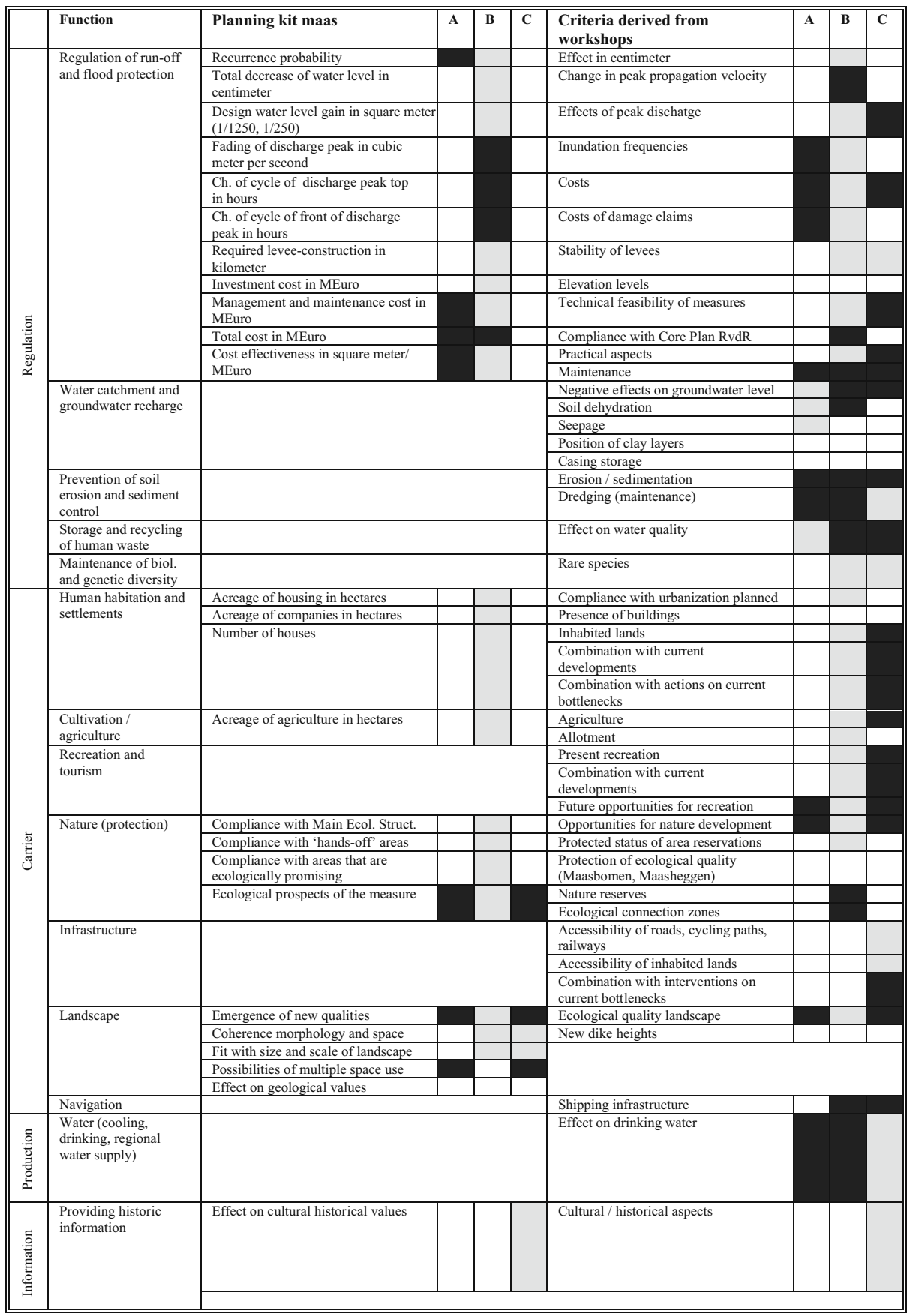

$A$ Temporal scale, $B$ spatial scale, $C$ constr. scale, white small/low, grey medium/intermediate, black long/large/high. 
here). Cost is still a contextualized variable, and a specific property of the 'flood mitigation strategy', and hence a low construal level applies.

- Cost effectiveness: derived from the above, but translated back to individual measures. Some measures are more cost effective than others, and due to the relation to individual measures a medium spatial scale applies. The other characteristics are the same as for the previous variables.

\subsubsection{Nature}

- Compliance with Main Ecological Structure (Ministerie van LNV (Ministry of Agriculture, Nature protection and Food safety), 1990): represents the overlap of proposed measures with areas that have been indicated as ecological zones. As such the Ministry regards the status quo (small temporal scale) over a regional area. The regional area implies a medium spatial scale. Since the policy guideline indicates the protected areas, the compliance with it can be characterized as a very concrete and contextualized bit of information, so a low construal level applies.

- Compliance with 'Hands-off' areas (Ministerie van Verkeer en Waterstaat and Rijkswaterstaat Dienst Limburg (Ministry of Traffic, Public works and Water management), 2003): similar to the previous indicator

- Compliance with areas that are ecologically promising: similar to the previous.

- Ecological prospects of the measure: the ecological prospects depend on the long term ecological development scenario applied. Hence this variable needs assessment on a large temporal scale. The spatial scale can be regional, which is reasonable when taking into account that the ecological development will strongly depend on the development of other functions, such as urbanization. The ecological prospect as such is a rather general description of a future state. It is not easily contextualized due to the long time horizon applying and therefore considered to be a high level construal.

\subsection{Indicators Used in Stakeholders' Argumentation}

In the second phase of the project (IVM-2), local and regional stakeholder affiliations discussed the proposed measures in a series of workshops. The objective of this second phase was to assess the proposed measures with the help of local and regional parties. In the beginning of this process the assumptions that underlay the project (climate change leads to higher peak discharges, which pose an actual threat that could be mitigated by taking the proposed measures) were not shared by all stakeholders. After discussing these assumptions, all stakeholders came to the general agreement that increasing peak discharges will indeed pose a threat to the catchment, and the discussion addressed the proposed measures.

An overview of the reported indicators and an assessment of their nature is given in Table 3. For purposes of objectivity, the formal reports of the first series of meetings were followed to derive the indicators (Ministerie van Verkeer en Waterstaat and Rijkswaterstaat Dienst Limburg (Ministry of Transport, Public Works and Water Management) 2004/2005). Where clarifying, personal workshop notes have been added. The assessment takes place in a similar manner as in the previous section. That means that the variables which came up are linked to the 
processes they relate to. Again, for clarification of the comparison, only 'regulation of run-off and flood protection' and 'nature' are described.

\subsubsection{Regulation of Run-off and Flood Protection}

- Effect in centimeter: follows from a comparison of the maximum water level in the current situation compared to that after measures have been implemented. The water levels are calculated based on peak discharges, taking place in cycles of a couple of days. The temporal scale is small. The decrease of water levels depends on the location and type of measure, and the length of backwater curves. Taking the scale of the catchment into account a medium spatial scale is assigned. The decrease of the water level in $\mathrm{cm}$ depends on local circumstances and is meaningless without this context. It is also a subordinate indicator, and considered a low level construal.

- Change in peak propagation velocity: relates to traveling of the discharge peak through the catchment. This process is usually described over a period of several days; a small temporal scale applies. The spatial scale is large, because the majority of the catchment has to be taken into account. Again the variable is described on a low construal level.

- Effects of peak discharge: this refers to an evaluation not of the measures, but of the effects in the current situation without measures being implemented: 'The threat is not so big as people say. High discharges will at most lead to nuisance and inconvenience, they pose no real danger' (Janssen 2004a). Apparently, the stakeholders find the local effects in the current situation important for their assessment of the proposed measures. It here concerns evaluation of the status quo, combined with the conveyance of a high discharge, so a small temporal scale applies. Because the effect is local, the applying spatial scale is medium. It is however not clear which effects the stakeholders are exactly referring to; the indicator stated is superordinate in nature and poses a general comment on peak discharges. It is considered a high level construal.

- Inundation frequencies: have to be addressed on a relatively large temporal scale, of over a decade. For zoning, stakeholders want to know what the expected inundation frequency of different areas is, in order to be able to assess the extent to which a measure can be combined with existing or newly developed functions. The inundation frequency pertains to relatively small areas (comparable to measure scale), so a medium spatial scale applies. The inundation frequency can be regarded a low level construal, since it is subordinate and a specific characteristic.

- Costs: during the stakeholder discussion the cost aspect came up as well, although it remained unclear what costs were referred to exactly. Apparently, people tended to refer to the costs of measures. From the discussion it becomes clear that the costs were considered in a more general way here than they were in the model; 'The cost of measures should not exceed the damage that is possibly caused by not taking them' (Janssen 2005). 'Who is going to pay for all these measures anyway? If it's not me, I don't mind them being more expensive' (Janssen 2004b). The stakeholders involved a cost-benefit point of view and a 'who is paying' question. The criterion 'costs' hence became more general and superordinate. Although the same time scale (including maintenance) and spatial 
scale (based on individual measures) apply as in the model, the costs as referred to by the stakeholders are an example of a high level construal.

- Costs of damage claims: refer to damage as an effect of flooding. To obtain a balanced figure here, the probability of the flood event has to be taken into account, meaning that a large time scale applies. The damage can be local in nature, so medium spatial scale is assigned. The cost of damage claims strongly relates to the value of property, a contextualized and specific characteristic of 'flood catastrophe', and is a low level construal.

- Stability of levees: pertains to the status quo. Is generally assessed on a local, medium spatial scale. The stability of levees says something about the current flooding probability, but is not entirely subordinate because diverse failure mechanisms apply. Because more concrete characteristics are needed to fill this criterion in (i.e. these failure mechanisms), a medium construal level applies.

- Elevation levels: underlie the inundation frequencies. This property can vary strongly over space (small spatial scale) and assumes the status quo as a starting point (small timescale). Higly subordinate and concrete, so low construal level.

- Technical feasibility of measures: static variable (unless one takes into account the technological development over time, but this is very hard to anticipate). The technical feasibility depends also on characteristics of the area in which the measure is to be implemented, so a medium spatial scale is assigned. The technical feasibility, however, remains a very abstract and general concept and is considered a high level construal.

- Compliance with the Core Planning Decision Room for the River (in Table 3 referred to as Core Plan RvdR): like compliance with other policy guidelines, this refers to an evaluation of the status quo. The guideline concerns the whole river, so a large spatial scale. Compliance with the guideline is a low level construal.

- Practical aspects: relates to the way in which a measure can be fitted into the current (infrastructural) situation. The characterization is the same as for technical feasibility.

- Maintenance: again, the stakeholders opted for a broader definition of maintenance than just the costs, which were used in the model. They also refer to the degree of sedimentation or erosion in other parts of the river bed, and the longterm development of maintenance policy. The temporal and spatial scales are large. Due to the broader implications and the more general formulation, the construal level is high.

\subsubsection{Nature}

- Opportunities for nature development: involves the expected future ecological development of the area. This criterion is assessed similar to 'ecological prospects of the measures' in Section 4.1.

- Protected status of area reservations: reservation of area for river measures induces limitations of other functions to that area. Some stakeholders reason that e.g. retention zoning allows for nature development, since other functions will no longer be allowed. In some cases this can be an advantage for the development of nature. The assessment of this indicator depends on the measure, reasoning from the current situation. The effects in terms of this status are concrete, and it is a specific effect of some measures; the criterion is considered a low level construal. 
- Protection of ecological quality: follows the same reasoning as the above, but now starting from existing ecological values. These can be very local in nature, so here a small spatial scale applies. Stakeholders mentioned characteristic types of vegetation as examples of ecological quality to be protected.

- Nature reserves: assignment of characteristics similar to "compliance with main ecological structure' in Section 4.1, but in general concerns larger areas.

- Ecological connection zones: indicating areas that provide connected habitats to all sorts of species. Assignment of characteristics follows the reasoning of 'nature reserves'.

\subsection{Comparison of Indicators Used by Modelers and Stakeholders}

Now that the indicators of model and stakeholders have been described in terms of the framework (Table 3), they can be compared. The comparison of the river functions is qualitative. For the comparison of temporal and spatial scale and level of construal, a Chi square test was applied to explore to what extent the indicators in the model differ in characteristics of those that were put forward by the stakeholders. We have to remark that there is an ongoing dispute about the applicability of this test to small sample sizes, such as occasionally occur in this study. We still assume that the outcomes will at least give an indication of the resemblance between the two classes. For the comparison of temporal and spatial scale and level of construal, a Chi square test was applied to explore to what extent the indicators in the model differ in characteristics of those that were put forward by the stakeholders. The number of model indicators with a certain class/property combination (e.g. for the function 'regulation of run-off and flood-protection' the 'long temporal scale' occurred four times) was used as a basis for the calculation of the 'expected probabilities'. The numbers of each combination as counted in the list of workshop indicators as 'observed values' (in this case, for the same function the long temporal scale occurred four times as well). The Chi square test is defined by formula 1.

$$
\chi^{2}=\sum_{i=1}^{n} \frac{\left(O_{i}-E_{i}\right)^{2}}{E_{i}}
$$

With:

- $\quad E_{i}$ the expected value based on the distribution of model indicators over the different possible classes (low, medium and high) per property (temporal scale, spatial scale and construal level). Each property has its own Chi square value.

- $O_{i}$ the observed occurrences for every combination in the stakeholder indicators.

The frequencies of occurrence of class/property combinations (e.g. for the function 'regulation of run-off and flood-protection' the 'long temporal scale' occurred four times) were taken as 'expected probabilities', and the frequencies derived from the workshop indicators as 'observed values' (in this case, for the same function the long temporal scale occurred four times as well).

The critical value with two degrees of freedom and $p=0.05$ is $\chi^{2}=5.99$. Values exceeding this value indicate that it is likely that the distributions differ.

The comparison was made only for the functions that are represented in both model and workshops, to obtain a balanced comparison. This means that the stakeholders' indicators on e.g. 'water catchment and groundwater recharge', 'prevention 
of soil erosion and sediment control' and some other stakeholder indicators are not taken into account because they are not described in the model and can hence not be compared to it.

From the listing of output variables and workshops arguments, it appears that the model addresses less river functions than the workshop participants did. This is in agreement with the expectation that stakeholder participation fosters horizontal integration, i.e. integration with the inclusion of multiple aspects from different 'interests', disciplines or functions. The obvious explanation for the model containing less functions, is that the model is, by definition, a simplification of reality. Here the trade-off between the complex real world and the concessions which have to be done from a model point of view become apparent.

The temporal scales of the indicators differ. The Chi square test on the temporal scales shows that the differences between workshops and model are not significant $\left(\chi^{2}=0.4\right)$. Both the model and the stakeholders focus mostly on processes pertaining to short time scales or on the current situation. Stakeholders show a large interest in the combination of measure implementation with ongoing projects, for instance on planned nature, housing, or river engineering works. Apparently, the 'political momentum' plays an important role in the stakeholder acceptance of the proposed measures in the IVM case study.

For the comparison of the spatial scales the difference in distribution between the workshops and the model is also not significant $\left(\chi^{2}=1.4\right)$. From Table 3 it appears that both stakeholders and model show a slight preference for the intermediate spatial scale of 100-1000 m. This preference is expected to be prompted by the nature of the case-study; the focus is on the 'measure-scale', even though the underlying safety problem relates to a 'strategic', and thus catchment, scale. Large spatial scales, appropriate for the evaluation of river strategies rather than individual measures, also appear quite frequently. Small spatial scales only appear in a number of instances, and more in the stakeholder set than in the model set. Even though the problem at hand is in its' explorative phase (so no final plans are supposed to result from this process), some people draw the link to their own 'backyard situation', thereby bringing up indicators relating to the eventual implementation of measures, in the current environment and infrastructure.

The level of construal shows the largest difference between the model and the workshop indicators with $\chi^{2}=21$. Closer inspection shows that this is particularly due to a much larger number of high level construals in the stakeholder indicators than in the model indicators. In the model, the indicators are in general formulated in a more specific manner. For stakeholder understanding it seems important to make an effort to translate the variables back to broader and more general concepts which are more easily understood. In everyday life, people are not dealing with the specific (concrete) and exceptional types of system behavior, but rather with the more general (abstract) behavior and the core features of the system.

Figure 4 schematizes the stakeholders' indicators for the function 'nature', as assessed on the three dimensions. Some of the indicators have overlapping assessments, and are assigned to the same block in the figure. The indicators depicted are:

1. Opportunities for nature development (long time scale, average spatial scale and high construal level)

2. Protected status of area reservations (short time scale, average spatial scale, low construal level) 


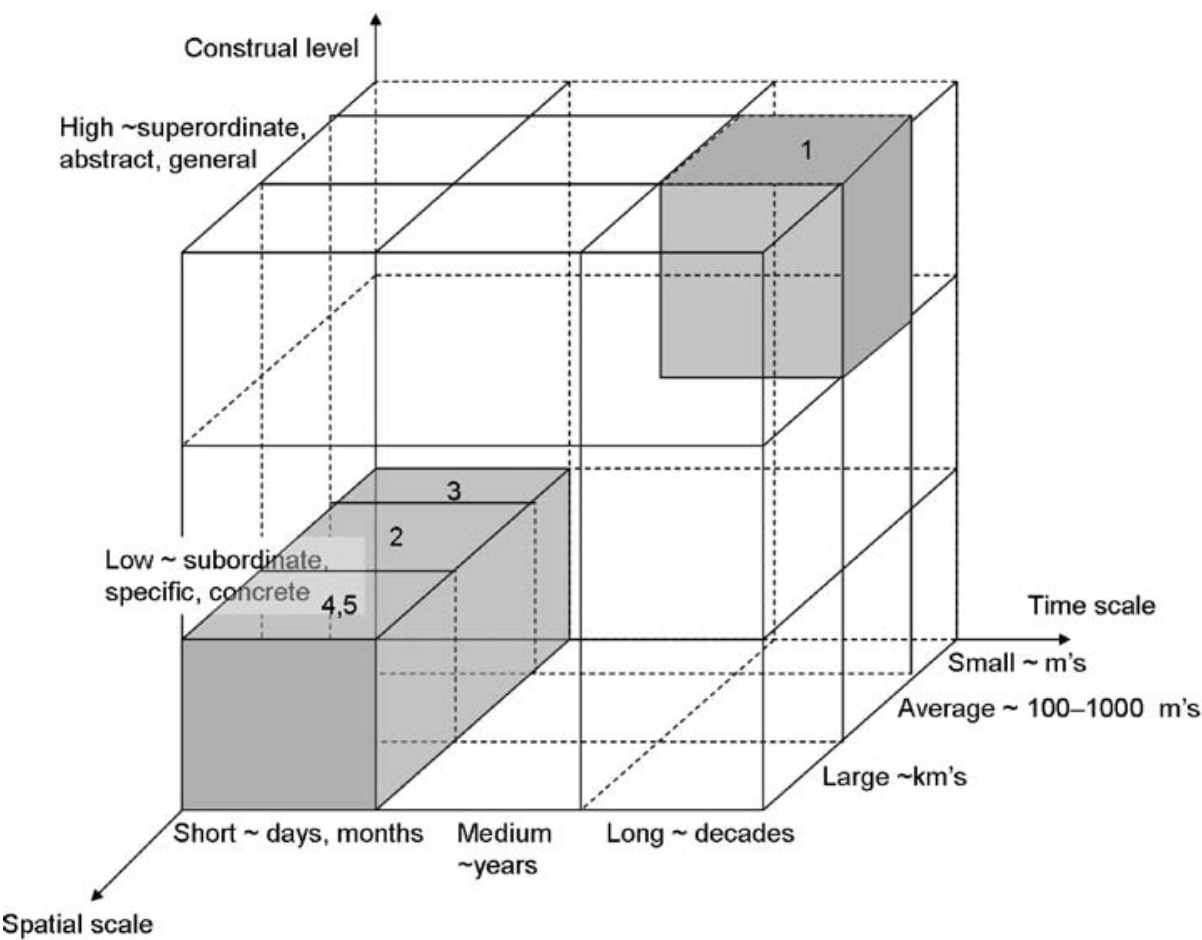

Fig. 4 Typology of stakeholders' indicators of 'nature'

3. Protection of ecological quality ${ }^{1}$ (short time scale, small spatial scale and low construal level)

4. Nature reserves (short time scale, large spatial scale, and a low construal level)

5. Ecological connection zones (same as (4)).

In Fig. 5 a cross section of the model is added, based on the model variables for the 'nature' function in the case study. The cross section represents a model scope, in this case capturing all time scales and levels of construal at an intermediate spatial scale. In the actual Planning Kit, only blocks 1 and 2 were included. The example model defined by the cross section is already more comprehensive. The cross section is chosen to illustrate the fact that in modeling, choices have to be made about the temporal and spatial scale and about the levels of construal addressed. Thus 'blanks' are revealed, where the required information is too abstract to be modeled, or where physical descriptions are lacking. The fact that not the entire spectra of all three features (leave alone for all possible functions) can be captured, results from the requirements and restrictions of modeling as summarized in Section 3.1. Indicators 3,4 , and 5 in Fig. 5 cannot be included if the model is based on the current choice of scales and construal levels.

\footnotetext{
${ }^{1}$ In this case, the ecological quality relates to the Maasbomen and Maasheggen areas, which are local ecological values when compared to the scale of rive reconstruction measures. They are hence assigned a small spatial scale.
} 


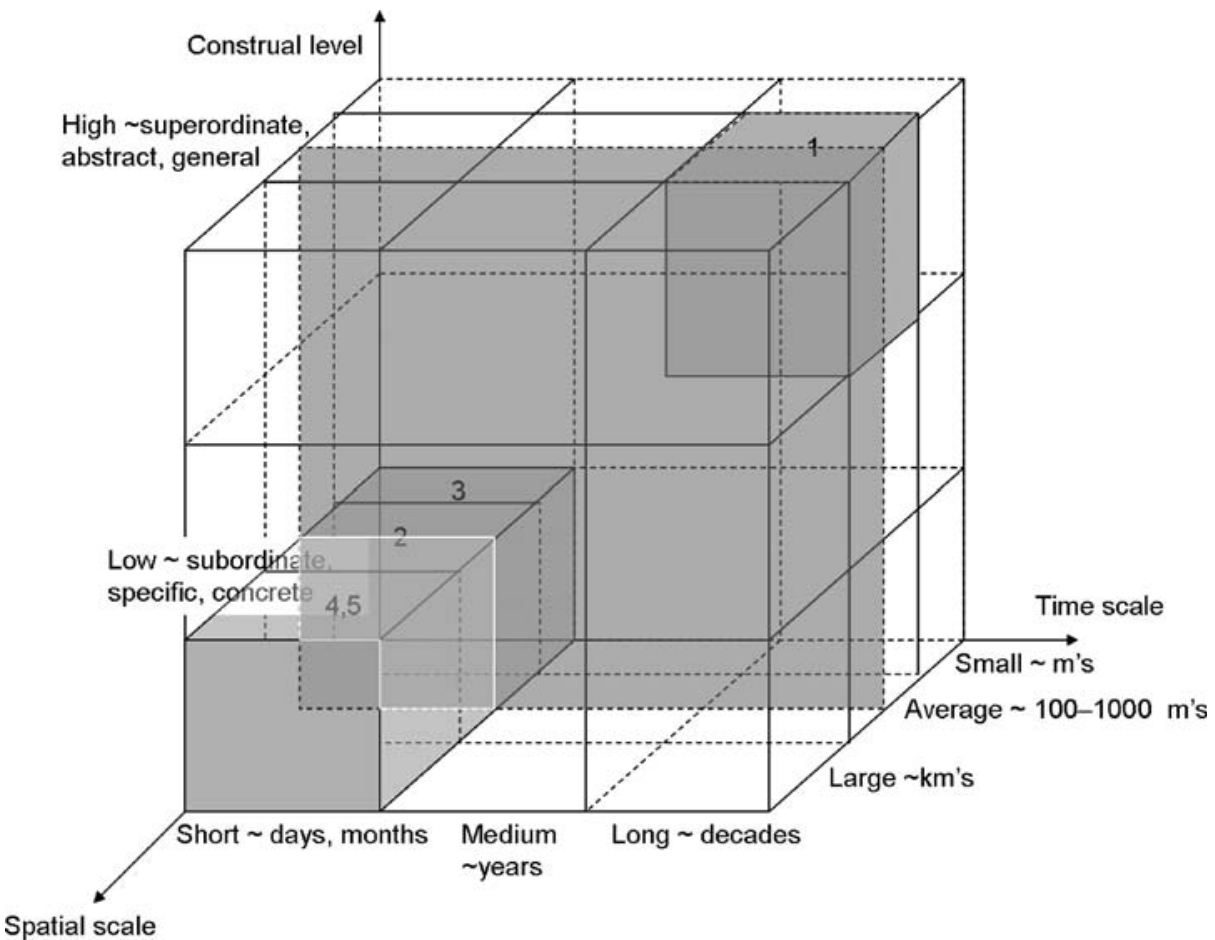

Fig. 5 Typology of stakeholders' indicators of 'nature' and model cross-section for 'nature'

Besides these characteristics, another choice made in modeling is that of the functions included-among other things depending on the purpose of the model. Approaches aiming at the inclusion of as many characteristics as possible will in general be based on building additional modules into the model, or on aggregation or disaggregation of data. It should be clear, however, that a fully integrated model as suggested by the definition of among others Pahl-Wostl (2004), combining all possible content-aspects, is not feasible. The more relations and the more complexity is introduced (i.e. more different cross-sections of the information-characteristicscube'), the more time- and money-consuming the modeling becomes. Moreover, the availability of data or mathematical relations for the different processes is usually limited. The framework presented here can help structuring the information needed concerning a certain problem to help optimize the utility of the modeling efforts. It also helps people in a policy process to determine to what extent modeling is the appropriate method to obtain the required information, and to what degree a model could be able to live up to their expectations.

\section{Discussion and Conclusions}

The framework provided in this paper provides a structured approach to information analysis in policy processes. Construal level theory makes the framework equipped to describe different perceptions which may play a role in river management processes. 
By merging relevant technical (river functions, temporal and spatial scale) and social features (construal levels) a more comprehensive understanding of the role of information in the policy process is obtained. By doing so, it helps understanding why people in such a process often perceive a 'gap' between themselves, and others in the process. Denominating the indicators in a model in terms of the framework helps showing which questions can and cannot be addressed with the model. It therefore shows people in the policy process (including the modelers themselves) which information needs to be addressed in a different manner (than with models), or with additional models. It supports realistic expectations of the applicability of models in the policy process and the integration of different types of information.

The classification of indicators along the four described dimensions will always take place in relation to the problem at hand. In our case, the problem is the strategic exploration of river management strategies. The strategies consist of measures. The assessment focuses on the measures; they are therefore considered to represent the 'average' spatial scale. For the other dimensions in the framework similar considerations play a role. This also means that depending on the model purpose in the policy process (see e.g. Van Daalen et al. 2002; Brugnach and Pahl-Wostl 2007), the overview of the indicators may work out different. This is not necessarily a problem, because the set of indicators will also be different for a different kind of problem. The framework is always applied in the context of the problem at hand.

From applying the framework to the Explorative Study of the Maas (IVM-2), a number of conclusions can be drawn:

- For modeling, the requirements of relevance, measurability, data-availability and simplicity are important restrictions. Modeling efforts will never succeed in providing all the necessary information in a river management process, simply because too many questions can be asked. Models can only provide part of the information used in a policy process. According to the evaluation of the IVM case study, this part is confined because only a limited number of river functions can be accounted for, and because there is a major focus on lower level construals (concrete, subordinate and specific pieces of information). In the IVM case, the involvement of stakeholders has led to a broader orientation in the decision making process (more river functions were accounted for) and the involvement of more abstract, superordinate information concerning the problem at hand. The discussion was literally brought to 'a higher level'. At the same time, expert information contributed to a well-informed decision. Different types of information are needed, and different tools are required to provide this information.

- The more resources become available, the more temporal and spatial scales can be linked in modeling, for instance by linking different calculation modules. Addressing additional river functions or higher level construals calls for innovative approaches towards modeling, able to work with more abstract (and hence often uncertain and qualitative) information. In as far as such approaches have not been developed or are not possible, other policy tools need to be utilized, such as workshops or discussions. The trade-offs made on the highest levels of construal essentially remain a topic of debate among stakeholders, experts and policy makers.

- By describing the different types of information in the policy process, the modeling effort can be more accurately deployed in the early stages of this 
process. At the same time, the stakeholder expectations of models can be tempered where necessary. This necessity stems from the restrictions mentioned above. The framework helps outlining a possible 'gap', and thus suggests also where people involved in the process will have to find a compromise. When discussing river strategies for instance, the use of small spatial scales may well be superfluous.

Acknowledgements The funding of this research by the Cornelis Lely Foundation is thankfully acknowledged. The valuable discussions with Anne Wesselink and Maarten Krol from the University of Twente, that helped shaping some of the ideas presented in this paper, are greatly appreciated. We further acknowledge the comments by two anonymous reviewers.

Open Access This article is distributed under the terms of the Creative Commons Attribution Noncommercial License which permits any noncommercial use, distribution, and reproduction in any medium, provided the original author(s) and source are credited.

\section{References}

Blöschl G, Sivapalan M (1995) Scale issues in hydrological modeling: a review. In: Kalma JD, Sivapalan M (eds) Scale issues in hydrological modeling. Wiley, Chichester, pp 9-48

Borowski I, Hare M (2007) Exploring the gap between water managers and researchers: difficulties of model-based tools to support practical water management. Water Resour Manage 21:10491074. doi:10.1007/s11269-006-9098-z

Brandon PR (1998) Stakeholder participation for the purpose of helping ensure evaluation validity: bridging the gap between collaborative and non-collaborative evaluation. Am J Eval 19: 325-337

Brugnach MA, Pahl-Wostl C (2007) A broadened view on the role for models in natural resource management: implications for model development. In: Pahl-Wostl C, Kabat P, Möltgen J (eds) Adaptive and integrated water management. Coping with complexity and uncertainty. Springer, Heidelberg, pp 184-203

Brugnach M, Tagg A, Keil F, de Lange WJ (2006) Uncertainty matters: computer models at the science-policy interface. Water Resour Manage 21:1075-1090. doi:10.1007/s11269-006-9099-y

Busch S (ed) (2004) Karakterisering stroomgebied Maas (Nederland) conceptual report Aug 2004. Regionaal Bestuurlijk Overleg Maas, Rotterdam

Dale VH, Beyeler SC (2001) Challenges in the development and use of ecological indicators. Ecol Indic 1:3-10. doi:10.1016/S1470-160X(01)00003-6

De Groot RS (1992) Functions of nature-evaluation of nature in environmental planning, management and decision making, $\mathrm{PhD}$ thesis, Wageningen Agricultural University, Wageningen

De Kok J-L, Wind HG (eds) (2003) Design and application of decision support systems for integrated water management; lessons to be learnt. Phys Chem Earth 28:571-578 (incorporating part A, B, and $\mathrm{C}$ )

Evans TP, Ostrom E, Gibson C (2003) Scaling issues in the social sciences. In: Rotmans J, Rothman DS (eds) Scaling in integrated assessment. Swets and Zeitlinger, Lisse

Gibson CC, Ostrom E, Ahn TK (2000) The concept of scale and the human dimensions of global change: a survey. Ecol Econ 32:217-239. doi:10.1016/S0921-8009(99)00092-0

Janssen, JAEB (2004a) Workshop Oss: personal notes of the IVM workshop, Oct. 21

Janssen JAEB (2004b) Workshop Sittard: personal notes of the IVM workshop, Sept. 30

Janssen JAEB (2005) Workshop Venlo: personal notes of the IVM workshop, Dec. 2

Liberman N, Trope Y (1998) The role of feasibility and desirability considerations in near and distant future decisions: a test of temporal construal theory. J Pers Soc Psychol 75:5-18. doi:10.1037/ 0022-3514.75.1.5

Lorenz CM, Gilbert AJ, Cofino WP (2001) Environmental auditing-indicators for transboundary river management. Environ Manage 28:115-129. doi:10.1007/s002670010211

Matthies M, Giupponi C, Ostendorf B (2007) Environmental decision support systems: current issues, methods and tools. Environ Model Softw 22:123-127. doi:10.1016/j.envsoft.2005.09.005 
McLain RJ, Lee RG (1996) Adaptive management: promises and pitfalls. J Environ Manage 20:437448. doi:10.1007/BF01474647

Ministerie van LNV (1990) Natuurbeleidsplan. Regeringsbeslissing. Tweede Kamer, vergaderjaar 1989-1990, 21149, nr. 2-3,'s-Gravenhage (in Dutch)

Ministerie van Verkeer en Waterstaat, Rijkswaterstaat Dienst Limburg (2003) Integrale verkenning Maas, Advies, Hoofdrapport en Achtergronddocumenten (cd-rom) sl (in Dutch)

Ministerie van Verkeer en Waterstaat, Rijkswaterstaat Dienst Limburg (2004/05) Conceptuele verslagen van de eerste serie workshops, Sittard, Venlo, Roermond en Den Bosch', RWS DLB, Maastricht (in Dutch)

Niemeijer D (2002) Developing indicators for environmental policy: data-driven and theorydriven approaches examined by example. Environ Sci Policy 5:91-103. doi:10.1016/S14629011(02)00026-6

Nieuwkamer RLJ (1995) Decision support for river management. PhD thesis, University of Twente, Enschede

Olsson JA, Anderson L (2007) Possibilities and problems with the use of models as a communication tool in water resource management. Water Resour Manage 21:97-110. doi:10.007/s11269006-9043-1

Otter H, Valkering P, Wolters H (2004) Participation and social learning in the Meuse River-case study reported under work package 5. Harmonicop, Delft

Pahl-Wostl C (2002) Towards sustainability in the water sector-the importance of human actors and processes of social learning. Aquat Sci 64:394-411. doi:10.1007/PL00012594

Pahl-Wostl C (2004) The implications of complexity for integrated resources management. In: Pahl-Wostl, C, Schmidt S, Rizzoli AE, Jakeman AJ (eds) Complexity and integrated resources management-transactions of the 2nd biennial meeting of the international environmental modeling and software society. Osnabrück

RIWA-Maas (2007) River Water Maas, international cooperation of drink water companies, www.RIWA-Maas.org. Cited July 32007

Trope Y, Liberman N, Wakslak C (2007) Construal levels and psychological distance: effects on representation, prediction, evaluation and behavior. J Consum Psychol 17:83-95

Trope Y (2004) Theory in social psychology: seeing the forest and the trees. Pers Soc Psychol Rev 8:193-200. doi:10.1207/s15327957pspr0802_13

Turnhout E, Hisschemöller M, Eijsackers H (2007) Ecological indicators: between the two fires of policy and science. Ecol Indicators 7:215-228

Van Daalen EC, Dresden L, Janssen MA (2002) The role of computer models in the environmental policy life cycle. Environ Sci Policy 5:221-231. doi:10.1016/S1462-9011(02)00040-0

Van der Veen A, Otter H (2003) Scales in economic theory. In: Rotmans J, Rothman DS (eds) Scaling in integrated assessment. Swets and Zeitlinger, Lisse

Wakslak CJ, Trope Y, Liberman N, Alony R (2006) Seeing the forest when entry is unlikely: probability and the mental representation of events. J Exp Psychol Gen 135:641-653. doi:10.1037/ 0096-3445.135.4.641

Walker DH (2002) Decision support, learning and rural resource management. Agric Syst 73:113127. doi:10.1016/S0308-521X(01)00103-2

Wesselink A (2006) Verkenningen hoogwaterbeheer Maas; een studie naar besluitvorming als verweving van expertise en belangen (Explorative Study of flood protection along the Maas: merging expertise and interest). Civil Engineering and Management research report 2006R-003/ WEM-004. University of Twente, Enschede (in Dutch)

World Bank (1999) Environmental performance indicators. A second edition note. World Bank, Washington DC 